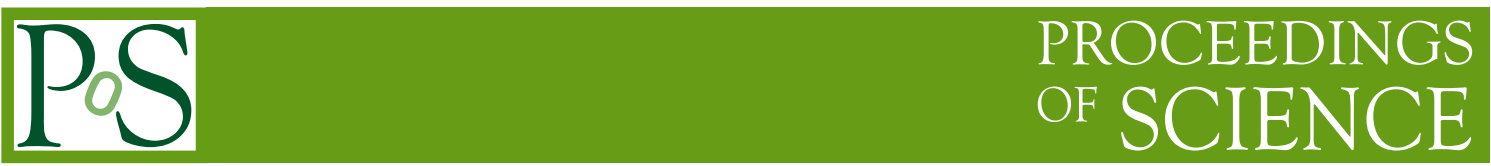

\title{
Quantum entanglement and KPZ relations
}

\section{Ferdinando Gliozzi*}

Dipartimento di Fisica Teorica, Università di Torino and

INFN, Sezione di Torino, Italy

E-mail: ferdinando.gliozzi@to.infn.it

\begin{abstract}
We point out that in quantum systems with many degrees of freedom the quantum entanglement of inaccessible regions generates a back-reaction which may modify the dynamics of the system. In particular we argue that in $1+1$ dimensional quantum systems described by conformal field theory (CFT) the back-reaction of the inaccessible subsystems has the same effect as the coupling to 2D quantum gravity. Numerical experiments on critical 2D Ising models show that the universality class is modified by such a back-reaction and that the new critical exponents agree with those predicted by the formula of Knizhnik, Polyakov and Zamolodchikov.
\end{abstract}

The XXVIII International Symposium on Lattice Filed Theory

June 14-19,2010

Villasimius, Sardinia Italy

${ }^{*}$ Speaker. 


\section{Introduction}

Long time ago Unruh [1] pointed out that, in the context of relativistic quantum field theory, an accelerated detector in flat spacetime should detect quanta even if the vacuum is in a pure state. This phenomenon comes from the fact that such a detector cannot see the whole spacetime: there is a region that classically cannot influence or be influenced by events outside it. To describe the quantum state as seen by such a detector, we should trace over the degrees of freedom in the unseen region of the spacetime. This generates a mixed state describing the detector measurements, with entropy -hence quanta- coming from the entanglement of the inaccessible region. Such an exchange of quanta between the accelerated detector and the inaccessible region of the spacetime defines a sort of interaction entirely generated by quantum entanglement. In this talk I would like to outline a general method to study this interaction in lattice field theory. This method is then applied to quantum spin chains described at criticality by a CFT. By applying renormalization group arguments one is led to conclude that the interaction produced by quantum entanglement is equivalent to the coupling of the system to $2 \mathrm{D}$ quantum gravity. This conclusion is supported by Monte Carlo calculations on critical Ising model, where the measured critical exponents, once this sort of interaction is switched on, turn out to agree with those predicted by the formula of Kniszhnik, Polyakov and Zamolodchikov in the context of quantum gravity.

\section{Reduced density matrix and Rényi entropy}

A complete description of the information available to an observer who has access only to a subsystem $A$ of a system in the fundamental state $|\Psi\rangle$ is encoded in the reduced density matrix

$$
\rho_{A}=\operatorname{tr}_{B}|\Psi\rangle\langle\Psi|
$$

obtained by tracing over the degrees of freedom of the complement of $A$, denoted with $B$, inaccessible to the observer. The quantities which are commonly used to measure the entanglement are expressed in terms of $\operatorname{tr} \rho_{A}^{n}$. For instance, the Tsallis entropy is $T_{A}(n)=\left(\operatorname{tr} \rho_{A}^{n}-1\right) /(1-n)$, the Rényi entropy is $R_{A}(n)=\log \operatorname{tr} \rho_{A}^{n} /(1-n)$ and the entanglement entropy can be written as the limit $S_{A}=\lim _{n \rightarrow 1} T_{A}(n)=\lim _{n \rightarrow 1} R_{A}(n)=-\operatorname{tr} \rho_{A} \log \rho_{A}$.

In quantum field theory (QFT) the quantity $\operatorname{tr} \rho_{A}^{n}$ for integer $n$ can be computed without explicit knowledge of the ground state through the so-called replica method [2, 3, 4]. Following this procedure the partition function $Z^{n}$ of $n$ copies (or replicas) of $d$ dimensional quantum system is computed in the standard way by doing the functional integration of the corresponding Euclidean classical system in $d+1$ dimensions. We have

$$
Z^{n}=\int \prod_{k=1}^{n} \mathscr{D}\left[\phi_{k}\right] e^{-\sum_{k=1}^{n} S\left[\phi_{k}\right]},
$$

where $\phi_{k}$ is a field configuration associated to the $k^{\text {th }}$ copy and $S[\phi]$ is the Euclidean action; $\operatorname{tr} \rho_{A}^{n}$ can be written as the vacuum expectation value of a suitable observable $\mathbb{O}$ defined on this larger system composed of $n$ replicas of the original system. The subsystem $B$ establishes a process of transferring information among the $n$ replicas through a specific coupling: the lattice links coming out of nodes of $B$ and directed into the (Euclidean) time direction $\tau$ cyclically connect the copy $k$ 
with the copy $k+1[3,4]$. Let us denote with $S_{B}\left[\phi_{1}, \phi_{2}, \ldots, \phi_{n}\right]$ the corresponding coupled action. It is easy to see that the quantity

$$
\mathbb{O}=e^{-\left(S_{B}^{(n)}\left[\phi_{1}, \phi_{2}, \ldots, \phi_{n}\right]-\sum_{k=1}^{n} S\left[\phi_{k}\right]\right)},
$$

has the desired property [5]. In fact its vacuum expectation value in the system of $n$ independent copies of the original system is

$$
\langle\mathbb{O}\rangle_{n}=\frac{\int \prod_{k=1}^{n} \mathscr{D} \phi_{k} \mathbb{O} e^{-S\left[\phi_{k}\right]}}{Z^{n}}=\frac{\int \prod_{k=1}^{n} \mathscr{D} \phi_{k} e^{-S_{B}^{(n)}}}{Z^{n}}=\frac{Z_{n}(A)}{Z^{n}}=\operatorname{tr} \rho_{A}^{n} .
$$

$Z_{n}(A)$ is the partition function of the system in the $n$ coupled replicas.

\section{The back-reaction of the inaccessible subsystems}

Contrarily to the usual approaches to quantum entanglement, we treat the accessible subsystem $A$ (or, equivalently, $B$ ) as a dynamical object that interacts with the rest of the system, so that its form and position is not constant in the time, but is determined by its interaction with the background. A way to implement this dynamics in a stationary system is to put it in equilibrium with the Gibbs' ensemble $\{A\}$ of all possible subsystems.

In lattice field theories the recipe is very simple. Each stack of $n$ links associated to the nodes $x$ and $y$ in the $n$ replicas is set in two possible states. In the state 'A' each link of the stack connects points of the same replica while in the state ' $\mathrm{B}$ ' it connects them cyclically; the links in the state ' $\mathrm{B}$ ' single out the subsystem $B$. It is sometimes convenient to to define a slightly generalized coupling among the $n$ replicas, relaxing the constraint of the $B$ subsystems to lie on a constant time slice and treating spatial and temporal links in the same way. An advantage of this more general setting is that it is easy to show that the coupled system of $n$ replicas is endowed with an important local symmetry: flipping from 'A' to 'B' or vice versa, the state of all links intersecting an arbitrary closed $d$ dimensional manifold keeps invariant the partition function [5]. A direct consequence of such a symmetry is that one can demonstrate that not only the entanglement entropy but all the thermodynamic functions depend only on the boundary of $B$ [5]. In order to promote accessible subsystems to dynamical variables one has simply to sum over all possible assignments of the states 'A' and 'B' to the lattice links, so the partition function of our coupled system of $n$ replicas can be written as

$$
Z_{n}=\sum_{\{G\}} \int \prod_{k=1}^{n} \mathscr{D} \phi^{(k)} e^{-S_{B}^{(n)}}
$$

where $G$ is the subgraph of links which are set in the state 'B' and the summation is over all subgraphs.

In this manner we obtain a general method which transforms whatever QFT theory in a new, non-trivial QFT generated by its interaction with the set of the possible non accessible subsystems. Being a completely general recipe one could suspect that the modified theory could describe the coupling of the original QFT with some universal interaction. We shall argue that in the case of 2D CFT such a modification describes its coupling to 2D quantum gravity. To such a purpose we specialize now to the case where the system in question is a quantum spin chain described at 


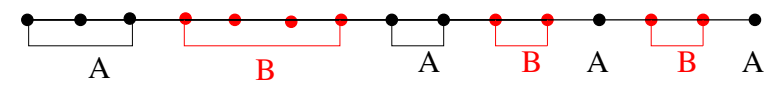

Figure 1: Partition of the spin chain in two arbitrary subsets $A$ and $B$.

criticality by a CFT. The subsystem $B$ is now an arbitrary subset of nodes of the chain (see Fig. 1); its two dimensional lattice description (3.1) is a discretized version of a $n$-sheeted covering of the plane, where the dual $\widetilde{G}$ of the subgraph $G$ is formed by the set of cuts connecting these sheets. The local symmetry mentioned above turns out to express the invariance of the system under the addition (or the removal) of closed cuts or under continuous deformations of open cuts with fixed ends. Then the sum over the subgraphs $G$ which select the inaccessible subsystems is equivalent to a special sum over the moduli and the genera of the Riemann surfaces described by the $n$ - sheeted covering of the plane. This is a first clue that the sum (3.1) over the ensemble of inaccessible subsystems is somehow related to $2 \mathrm{D}$ quantum gravity.

Another, much stronger, indication comes from the following renormalization group argument. In a CFT defined on a $n$-sheeted covering of the plane, the conical singularities located at the branch points are associated to primary fields $\Phi_{n}(z, \bar{z})$ of scaling dimensions [6]

$$
\Delta_{n}=\bar{\Delta}_{n}=\frac{c}{24}\left(1-\frac{1}{n^{2}}\right)
$$

where $c$ is the central charge. As a consequence, for not too large $c$ the conical singularities are relevant perturbations of the CFT. This means that, if we describe the critical system on the $n$ sheeted covering of the plane with the action

$$
S=S^{*}+\mu \int \Phi_{n}(z, \bar{z}) d^{2} z
$$

where $S^{*}$ is the CFT action describing the unperturbed critical system and $\mu$ is the chemical potential which controls the appearance of conical singularities, these relevant perturbations drive the system away form the critical point. According to (3.2) any CFT with $c>0$ is unstable whenever perturbed with $\Phi_{n}$. This fact, combined with the $c$ - theorem [7], restricts the renormalization group flows into fixed points with $c=0$. For generic values of $\mu$ these are trivial fixed points corresponding to massive theories. However for a suitable value of $\mu$ the system may be critical, as we shall see in the last section. Then in the CFT describing this infrared fixed point the net effect of the dynamical conical singularities associated to the inaccessible subsystems is the complete screening of the matter central charge. This is precisely what happens when CFT is coupled to 2D quantum gravity, i.e. the sum over the conical singularities with the rules dictated by the quest of equilibrium with the ensemble of the inaccessible subsystems has the same effect as the sum over geometries with the rules of quantum gravity.

\section{The KPZ relations}

The Knizhnik-Polyakov-Zamolodchikov (KPZ) formula [8, 9] establishes the relation between the bare $\left(\Delta^{o}\right)$ and the dressed $(\Delta)$ scaling dimensions of a primary field of a CFT when the theory 
is coupled to quantum gravity:

$$
\Delta^{o}=\Delta+\frac{\gamma^{2}}{4} \Delta(\Delta-1), \quad \gamma=\sqrt{\frac{25-c}{6}}-\sqrt{\frac{1-c}{6}},
$$

where $c$ is the central charge. In the minimal $(\mathrm{p}, \mathrm{q})$ models with $c=1-6(\mathrm{p}-\mathrm{q})^{2} / \mathrm{pq}$ the spectrum of the bare scaling dimensions $\Delta^{o}$ is very well known (see for instance Ref. [9]). A lesser known fact is that in these minimal models it is possible to establish a one-to-one correspondence $\Delta_{\ell}^{o} \leftrightarrow \ell$ between the allowed values of $\Delta^{o}$ and the integers $\ell$ which cannot be written as $\mathrm{p} s+\mathrm{q} t$ with $s$ and $t$ non negative integers. In other words, the integers $\ell$ form precisely the set of holes of the infinite sequence [10]

$$
\mathrm{p} s+\mathrm{q} t, \quad s, t=0,1,2,3, \ldots .
$$

For instance, in the critical Ising model, corresponding to $(3,4)$, the holes of $(4.2)$ are at $\ell=1,2,5$, corresponding to the identity operator with $\Delta_{1}^{o}=0$, to the spin operator with $\Delta_{2}^{o} \equiv \Delta_{\sigma}^{o}=\frac{1}{16}$ and to the energy operator with $\Delta_{5}^{o} \equiv \Delta_{\varepsilon}^{o}=\frac{1}{2}$. More generally we have

$$
\Delta_{\ell}^{o}=\frac{\ell^{2}-(\mathrm{p}-\mathrm{q})^{2}}{(\mathrm{p}+\mathrm{q})^{2}-(\mathrm{p}-\mathrm{q})^{2}}, \quad \Delta_{\ell}=\frac{\ell-|\mathrm{p}-\mathrm{q}|}{\mathrm{p}+\mathrm{q}-|\mathrm{p}-\mathrm{q}|}
$$

where in the second equality we used the KPZ formula (4.1). It is interesting to note that the gravitational dressing of the scaling dimensions leads to a new set of critical exponents which are still rational.

In order to support our conjecture that the back-reaction of the inaccessible subsystems in 2D quantum theories has the same effect as the coupling to 2D quantum gravity we have to prove first that the the system on the multi-sheet covering of the plane has at least a critical point and then that the critical exponents of the associated universality class are the gravitationally dressed ones defined in (4.3). We shall see it in the next section by a Monte Carlo simulation of the Ising model, where we expect $\Delta_{\sigma}=\frac{1}{6}$ and $\Delta_{\varepsilon}=\frac{2}{3}$.

\section{A Monte Carlo calculation}

We simulated a quantum spin- $\frac{1}{2}$ chain in a transverse field by using a $2 \mathrm{D}$ Ising model in a square lattice of size $L \times L$ at the self-dual point with $n=2, \ldots, 5$ replicas. We updated the Ising part of the model with a standard non-local cluster algorithm. In order to control the number of branch points we introduced a fugacity $z$ and the update of $\{A\}$ was obtained with a heat bath method. The main results are reported in [11].

In a first set of numerical experiments the ensemble $\{A\}$ was taken on a $1 \mathrm{D}$ slice and on the same slice we measured the spin correlators at a fixed distance $L / s$ with $s=4$ or $s=8$. Varying the total size from $L$ to $L^{\prime}=\lambda L$ and taking the ratio of these correlators we got a power law $\lambda^{-x}$ which allows to estimate the critical exponent $x$. Since $\{A\}$ affected only a one dimensional boundary of our system, this remained critical even at $z \neq 0$. A typical result of this calculation is reported in figure 2: as $z$ varies from 0 to $1, \lambda^{-x}$ drops from the expected value of the pure Ising model with $x=4 \Delta_{\sigma}^{o}$ to a new scaling dimension. According to the previous discussion, the expected value is the one suggested by KPZ formula. As sometimes it happens in critical systems, the observed 


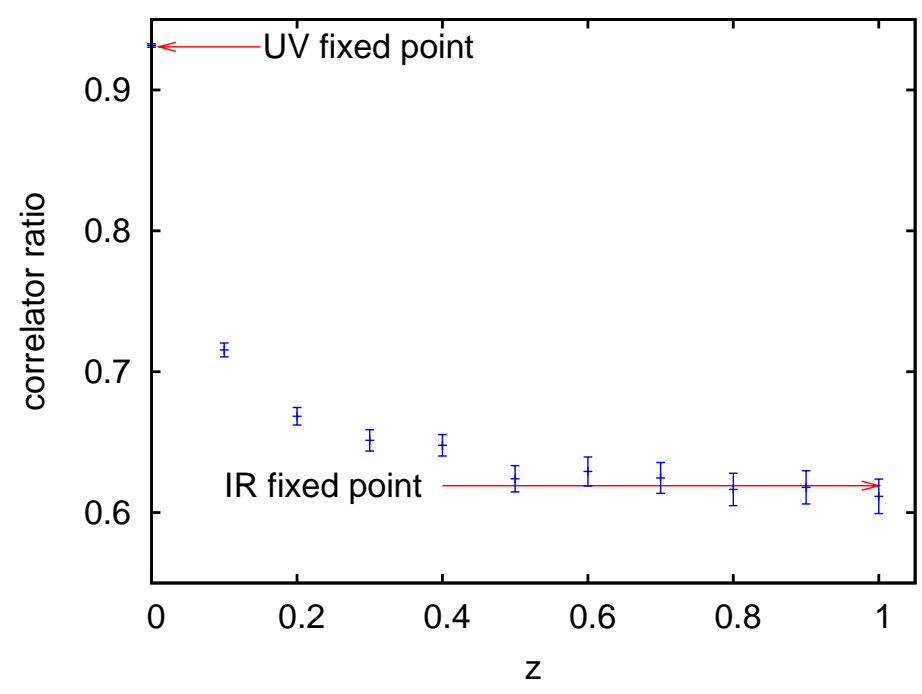

Figure 2: Plot of the critical ratio $\lambda^{-x}$ as a function of the fugacity $z$ in the 1D setup with two replicas. The two arrows represent the expected values for the pure system and the system coupled with the set of accessible subsystems.

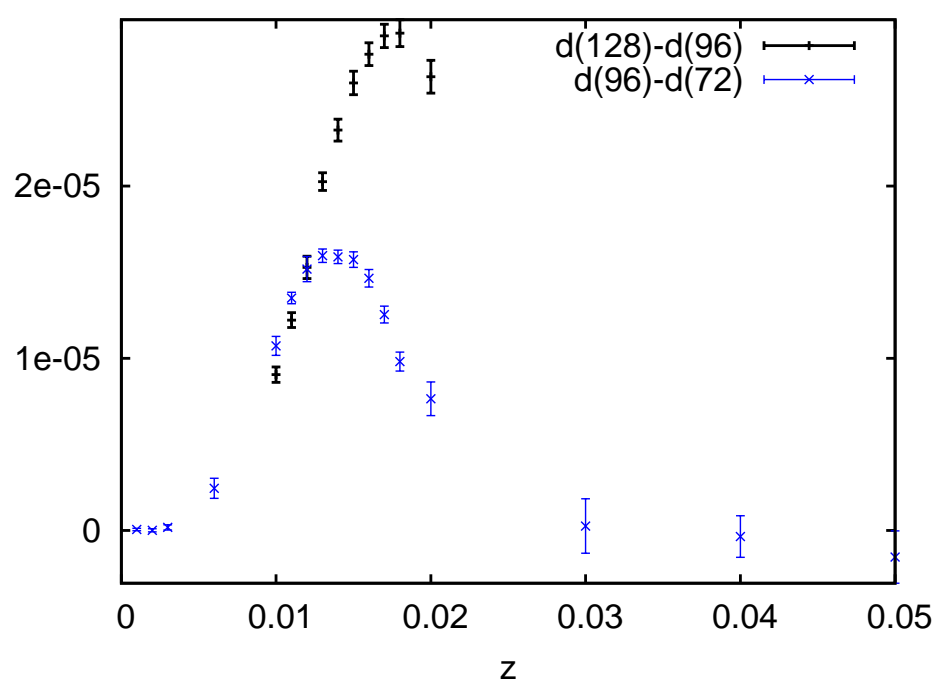

Figure 3: Plot of the difference of the density of conical singularities for lattice of different sizes. $d(L)$ is such a density in square box $L \times L$ in the case of two replicas. 
value of $x$ differs by an integer with respect to the expected value. Precisely we found $x=4 \Delta_{\sigma}+1$ for $n=2$ and $x=4 \Delta_{\sigma}+2$ for $n \geq 3$.

In a second set of numerical experiments we simulated instead a truly two dimensional system, with no limitations on the location of cuts representing the inaccessible subsystems. In this new setting the gas of conical singularities is spread in the bulk and drives the system away from the critical point of the pure system. There is however a critical value $z_{c}$ of the fugacity at which the system undergoes a second order phase transition which is presumably in the same universality class of the 1D quantum system described above. In order to locate such a critical value we studied finite size effects on the density of conical singularities. A typical plot of these finite size effects is drawn in figure 3. A finer estimation of $z_{c}$ was obtained by requiring a power-like behavior in the spin correlators. The final estimate was $z_{c}=0.01127(1)$. Measuring the spin correlator at this value of $z$ it turns out that the scaling dimension of the spin operator, within the numerical accuracy, the one of the 2D quantum gravity (see the figure 2 of [11]).

Likewise, the scaling dimensions of the energy operator turn out to be the KPZ value $\Delta_{\varepsilon}=\frac{2}{3}$. In order to extract this critical exponent, we measured the vacuum expectation value of the link operator $\langle$ link $\rangle$. This quantity is expected to have the following functional form at criticality

$$
\langle\text { link }\rangle=e_{0}+e_{1} / L^{2 \Delta_{\varepsilon}}+e_{2} / L^{2 \Delta_{\varepsilon}+1}+\ldots
$$

The fit parameters $e_{0}=1.42467(5), e_{1}=-3.94(7)$ and $e_{2}=257(5)$ fit accurately the data with a $\chi^{2} /$ d.o.f of 0.6 , as shown in figure 3 of [11].

In conclusion, we found that allowing the inaccessible subsystems to back-react has the same effect, in a 1D quantum system, as coupling it to 2D quantum gravity. The numerical algorithm used to update the geometry is new and it involves, besides a sum over the moduli of Riemann surfaces, also a sum over genera. This is very different from the standard appoach to 2D quantum gravity with dynamical triangulations, where the genus is kept fixed [12].

\section{References}

[1] W.G. Unruh, Phys. Rev. D 14 (1976) 870.

[2] C. G . Callan and F. Wilczek, Phys. Lett. B 333 (1994) 55.

[3] C. Holzhey, F. Larsen and F. Wilczek, Nucl.Phys. B424 (1994) 443.

[4] P. Calabrese and J. Cardy, J.Stat. Mech. (2004) P06002.

[5] M. Caraglio and F. Gliozzi, JHEP 0811 (2008) 076 [arXiv:0808.4094 [hep-th]].

[6] V. G. Knizhnik, Commun. Math. Phys. 112, 567 (1987).

[7] A. B. Zamolodchikov, JETP Lett. 43 (1986) 730 [Pisma Zh. Eksp. Teor. Fiz. 43 (1986) 565 ].

[8] V. G. Knizhnik, A.M. Polyakov and A. B. Zamolodchikov, Phys. Lett. A 3 (1988) 819.

[9] F. David, Mod. Phys. Lett. A3 (1988) 1651; J. Distler and H. Kawai, Nucl. Phys. B 321 (1989) 509.

[10] P. Dorey, C. Dunning, F. Gliozzi and R. Tateo, J. Phys. A 41(2008)132001 [arXiv:0712.2010[hep-th]]

[11] F. Gliozzi, arXiv:1006.4749 [hep-th].

[12] J.Jurkievicz, A. Krzywicki, B. Petersson and B. Soderberg, Phys. Lett. B 213 (1988) 511; C. F. Baillie and D. A. Johnston, Mod. Phys. Lett. A 7 (1992) 1519 [arXiv:hep-lat/9204002]. 